\title{
Opacity in the upper atmospheres of active stars
}

\section{AD Leonis}

\author{
D. J. Christian ${ }^{1}$, M. Mathioudakis ${ }^{1}$, D. S. Bloomfield ${ }^{1}$, J. Dupuis ${ }^{2}$, F. P. Keenan ${ }^{1}$, D. L. Pollacco ${ }^{1}$, and R. F. Malina ${ }^{3}$ \\ 1 Department of Pure and Applied Physics, The Queen's University Belfast, Belfast BT7 1NN, Northern Ireland, UK \\ e-mail: d.christian@qub.ac.uk \\ 2 Department of Physics and Astronomy, Johns Hopkins University, 3400 North Charles Street, Baltimore, MD 21218, USA \\ 3 Laboratoire d'Astrophysique de Marseille, BP 8, Traverse du Siphon, 13376 Marseille Cedex 12, France
}

Received 24 October 2005 / Accepted 5 February 2006

\section{ABSTRACT}

\begin{abstract}
We present FUV and UV spectroscopic observations of AD Leonis, with the aim of investigating opacity effects in the transition regions of late-type stars. The C III lines in FUSE spectra show significant opacity during both the quiescent and flaring states of AD Leonis, with up to $30 \%$ of the expected flux being lost during the latter. Other FUSE emission lines tested for opacity include those of O VI, while C IV, Si IV and N V transitions observed with STIS are also investigated. These lines only reveal modest amounts of opacity with losses during flaring of up to $20 \%$. Optical depths have been calculated for homogeneous and inhomogeneous geometries, giving path lengths of $\approx 20-60 \mathrm{~km}$ and $\approx 10-30 \mathrm{~km}$, respectively, under quiescent conditions. However path lengths derived during flaring are $\approx 2-3$ times larger. These values are in excellent agreement with both estimates of the small-scale structure observed in the solar transition region, and path lengths derived previously for several other active late-type stars.
\end{abstract}

Key words. atomic data - stars: activity - stars: atmospheres - stars: individual: AD Leonis - stars: late-type - ultraviolet: stars

\section{Introduction}

The transition region (TR) in late-type stars shows a temperature rise from the chromospheric value of a few $\times 10^{4} \mathrm{~K}$ to coronal temperatures of $\approx 10^{7} \mathrm{~K}$. In the solar case, the TR scale height has been constrained to $\leq 50 \mathrm{~km}$ (Doyle \& McWhirter 1980; Keenan \& Kingston 1986; Doschek et al. 2004). However such important information on the TR parameters are lacking for most stars. Several studies have investigated the TR scale heights of active stars, finding values of 10 to $100 \mathrm{~km}$ for active stars such as AU Mic (Bloomfield et al. 2002, Paper I), and the flare star Proxima Centauri (Christian et al. 2004).

Previous studies seeking to derive scale height for stellar TRs (Mathioudakis et al. 1999; Bloomfield et al. 2002; Christian et al. 2004) used the relation of optical depth to physical parameters, such as electron density and path length, and such a method is employed here. The optical depth at line center is given by:

$\tau_{0}=1.16 \times 10^{-14} \lambda f_{i j} \sqrt{\frac{M}{T}} \frac{n_{\mathrm{i}}}{n_{\mathrm{el}}} \frac{n_{\mathrm{el}}}{n_{\mathrm{H}}} \frac{n_{\mathrm{H}}}{n_{\mathrm{e}}} n_{\mathrm{e}} l$

where $\lambda$ is the wavelength in $\AA, f_{i j}$ the oscillator strength of the transition, $M$ the mass of the absorbing atom in atomic mass units, $T$ the temperature in $\mathrm{K}, l$ the path length in $\mathrm{cm}$ and $n_{\mathrm{i}}, n_{\mathrm{el}}$, $n_{\mathrm{H}}, n_{\mathrm{e}}$ the number densities in $\mathrm{cm}^{-3}$ of ions in the lower level $i$, element, hydrogen and free electrons, respectively (Mitchell \& Zemansky 1961; Jordan 1967). Atomic parameters for the transitions of the ions used in this work have been summarized in Christian et al. (2004).

The optical depths for particular emission lines are derived from ratios of line fluxes that are expected to have certain values in optically thin conditions, an assumed geometry, and the escape probability calculated for that geometry. For example, the C III $1175.71 \AA$ and $1174.94 \AA$ emission lines arise from a common upper level, and their expected line flux ratio is related to the ratio of their Einstein $A$-values. Under optically thin conditions, the ratio of the flux of the $\lambda \lambda 1175.71$ line to that of $\lambda \lambda 1174.94$ is expected to be 3 . Other transitions, such as those of O VI, N V, Si IV and C IV, have a common lower level and under optically thin conditions their expected line flux ratio scales as the ratio of their electron impact excitation rates, which for those lines is expected to be 2 . For a detailed description of the method used the reader is referred to Bloomfield et al. (2002).

In this paper we continue our in-depth study of opacity effects in stellar TR with an analysis of the well-known flare star AD Leonis (hereafter AD Leo, dM3.5e; BD+20²465, GJ 388). The high levels of activity and flaring behavior in AD Leo have made it a well studied candidate for flare-activity relations and it has been the subject of several multi-wavelength campaigns (Hawley et al. 2003). Emission lines arising from the transition region are observed to show significant downflows, in agreement with chromospheric condensation models applied to solar flares (Hawley et al. 2003). Studies of the coronal emission of AD Leo at X-ray wavelengths have found compact loop structures of size $\approx 30 \%$ of the star's radius (Favata et al. 1999; Sciortino et al. 1999).

The FUSE and HST observations and analysis of AD Leo are presented in Sect. 2. We derive light curves in two different emission lines from the FUSE observations and note that AD Leo was active with several flares. The temporal information is employed to selected spectra as a function of intensity, and we present the results of the spectral analysis in Sect. 3. From these spectra we derive ratios of line fluxes for emission features of interest, and these line ratios are used in Sect. 3.2 to calculate optical depths. The optical depths, combined with the values of 
the electron density, allow us to calculate the extent of the scattering regions, and we compare these results to those from other active late-type stars and the Sun in Sect. 4. Lastly, in Sect. 5, we present the conclusions of our findings and compare the results to the solar case and previous work on $\mathrm{AU}$ Mic, YZ CMi and Proxima Centauri.

\section{Observations and analysis}

FUSE observed AD Leo starting at 03:01 UT 2001 April 11 as part of program A022. The observations were made using the low resolution aperture (LWRS) in time-tagged mode and covered 41 satellite orbits for a total on source exposure time of $65 \mathrm{ks}$. A complete review of the FUSE telescope and instruments can be found in Moos et al. (2000) and Sahnow et al. (2000a,b). Briefly, FUSE consists of four co-aligned prime-focus telescopes, with two telescopes having $\mathrm{SiC}$ coatings and optimized for the 905-1105 $\AA$ region and the others using LiF coatings to cover $987-1187 \AA$. These channels cover important emission lines for our TR study, such as C III 977 and $1176 \AA$, and O VI 1032 and $1038 \AA$.

FUSE data were reduced with the latest available version (3.1.0) of the calfuse pipeline. The results were verified using the previous version of the pipeline (v2.4) and found in agreement. Spectra were extracted from the large aperture (LWRS) and these were background subtracted, flat-fielded, wavelength and flux calibrated as described in the calfuse reference guide (van Dixon et al. 2001). We have combined spectra from different detector channels to increase the signal to noise ratio. This was accomplished using the FUSE_REGISTER ${ }^{1}$ tool provided by the FUSE project. Combined spectra for the wavelength regions of interest are: segments $1 \mathrm{BSiC}$ and $2 \mathrm{ASiC}$ for C III $977 \AA$, segments $1 \mathrm{ALiF}$ and $1 \mathrm{ASiC}$ for O VI $1032 \AA$ and OVI $1038 \AA$, and segments $2 \mathrm{ALiF}$ and $1 \mathrm{BLiF}$ for the C III $1176 \AA$ A multiplet. All spectra were binned to a 4-pixel resolution which corresponds to $\sim 8 \mathrm{~km} \mathrm{~s}^{-1}$, approximately half of a formal resolution element.

Timing analysis of FUSE observations of AD Leo revealed a high level of activity with several flares. Light curves for the entire observations extracted in the lines of O VI $1032 \AA$ and the total C III $1176 \AA$ multiplet are shown in Fig. 1. We concentrated on the two largest flares with both complete rise and decay times (labeled 1 and 2), and extracted spectra from these flare intervals. Flare 1 had rise times $\leq 300 \mathrm{~s}$ for both O VI $1032 \AA$ and C III $1176 \AA$ multiplet light curves. However, the e-folding decay times for the flare were much shorter for C III, being $\approx 100 \mathrm{~s}$ while the decay time was nearly 1000 s for O VI. Similarly the rise times for flare 2 were $\approx 200$ s for both ions, and the flare decay time was $\approx 30$ s for C III and nearly 10 times longer for O VI. FUV flares observed in C III were also found to decay more rapidly for Prox Cen (Christian et al. 2004). The luminosities of flares 1 and 2 are $\approx 8 \times 10^{26} \mathrm{erg} \mathrm{s}^{-1}$ for the total flare in the C III $1176 \AA$ line and about 5 times higher for the flare peaks. These are about an order of magnitude higher than those observed for Prox Cen (Christian et al. 2004). The total flare 1 and 2 luminosities are $9.3 \times 10^{25} \mathrm{erg} \mathrm{s}^{-1}$ and $7.1 \times 10^{26} \mathrm{erg} \mathrm{s}^{-1}$ in the O VI $1032 \AA$ transition, respectively. The O VI $1032 \AA$ peak flare luminosities are approximately $50 \%$ higher.

We have also obtained archival Hubble Space Telescope Imaging Spectrometer (STIS) data to investigate important FUV lines which may also show opacity effects, such as

\footnotetext{
${ }^{1}$ http://fuse.pha.jhu.edu/analysis/fuse_idl_tools.html
}

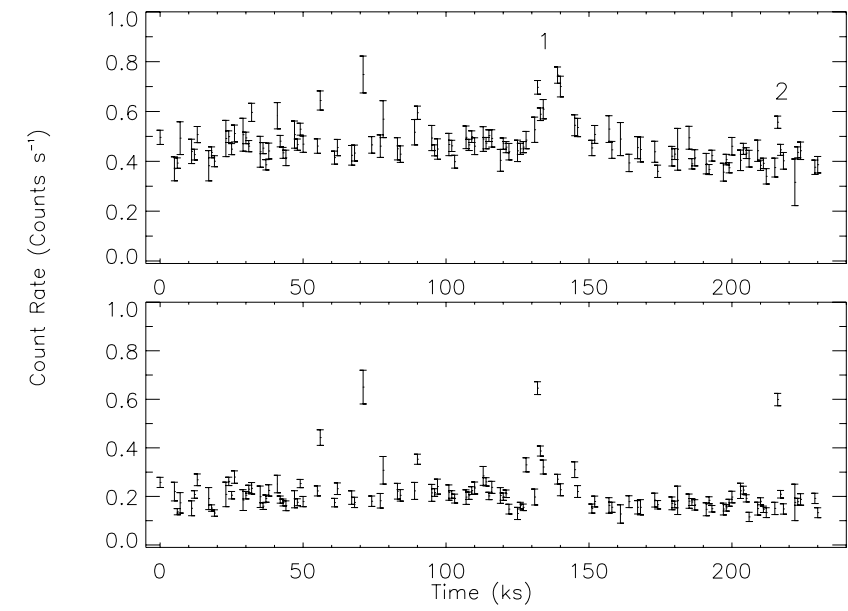

Fig. 1. AD Leo light curves for the entire FUSE observation. The top panel shows the O VI $1032 \AA$ light curve and bottom panel shows the C III $1176 \AA$ multiplet light curve. The start time for the first bin in MJD 52010.12598 and bin size is $1000 \mathrm{~s}$ for both light curves. Flares "1", and "2", are noted in the top panel.

C IV, Si IV and NV, as well as further measurements of C III. Additionally, O IV lines were used to constrain the electron densities. AD Leo was observed as part of a multi-wavelength campaign under HST program 8613 (root O61S; PI Hawley) on 2000 March 3. These observations were performed with the E140M grating covering $1150-1700 \AA, 0.2^{\prime \prime} \times 0.2^{\prime \prime}$ aperture, and FUV-MAMA detector, yielding a resolution of $\approx 70000$, and providing us with both quiescent and flaring spectra (Hawley et al. 2003). We also obtained the STIS observations of AD Leo on 2002 June 1 (program 9271; O6JG; PI A. Brown), which provided an additional quiescent spectrum. The 1-D extracted spectra were reduced and combined with the standard HST_CALIB.STIS IRAF routines. The O6JG found $\mathrm{AD}$ Leo in a quiescent state with an average flux of $1.67 \times$ $10^{-13} \mathrm{erg} \mathrm{cm}^{-2} \mathrm{~s}^{-1}$ (luminosity $=4.4 \times 10^{26} \mathrm{erg} \mathrm{s}^{-1}$ ) in the C III multiplet. However the earlier O61S observations found $\mathrm{AD}$ Leo to be very active. We created a total flare spectrum with a C III multiplet flux of $3.26 \times 10^{-12} \mathrm{erg} \mathrm{cm}^{-2} \mathrm{~s}^{-1}$ and a spectrum from the flare peak with a flux of $5.14 \times 10^{-12} \mathrm{erg} \mathrm{cm}^{-2} \mathrm{~s}^{-1}$. Our derived fluxes for the quiescent level of AD Leo were about $10 \%$ lower than those used by Hawley et al. (2003) for the O61S observation, and this reflects slightly different interval selections. The same holds for the largest AD Leo flare observed with STIS (Hawley's flare 8). Our selection of a larger flare interval has $\approx 50 \%$ less flux than the Hawley peak spectrum, but has similar line flux ratios.

As in our previous work (Bloomfield et al. 2002; Christian et al. 2004), we derived line fluxes by fitting Gaussians to the observed line profiles. In general, a single Gaussian profile was sufficient to fit the $\mathrm{O}$ VI and $\mathrm{C}$ III transitions. However, a small red component was detected in the profiles of some of the STIS N V, Si IV, and CIV lines and two Gaussians were fitted to these in both the quiescent and flaring spectra. The six component C III $1176 \AA$ multiplet was fitted with five separate Gaussians, with the $1175.59 \AA$ and $1175.71 \AA$ lines being treated as a single feature, and the central wavelength of each Gaussian was fixed relative to the $1174.9 \AA$ line (at the laboratory wavelength separation value), but the wavelength of the latter was left as a free parameter. In this way any shifts caused by the spectrometers could be taken into account. The Gaussian profile widths were 
Table 1. Line fluxes and ratios of the C III multiplet and O VI resonance lines observed from AD Leo with FUSE and STIS. The fluxes are given in units of $10^{-14} \mathrm{erg} \mathrm{cm}^{-2} \mathrm{~s}^{-1}$ followed by the estimated error in brackets. The fluxes given for each of the flares are the total observed fluxes minus the quiescent components.

\begin{tabular}{|c|c|c|c|c|c|c|c|c|c|c|c|c|}
\hline & \multicolumn{6}{|c|}{ 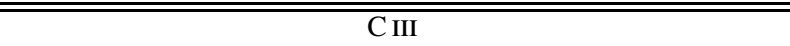 } & \multicolumn{6}{|c|}{ 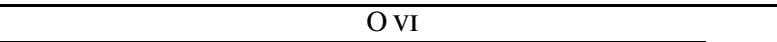 } \\
\hline & \multicolumn{2}{|c|}{ Flux } & \multirow[b]{2}{*}{ Ratio } & \multirow[b]{2}{*}{$\frac{P_{\text {thick }}}{P_{\text {thin }}}$} & \multicolumn{2}{|c|}{$\tau_{1175.7}$} & \multicolumn{2}{|c|}{ Flux } & \multirow[b]{2}{*}{ Ratio } & \multirow[b]{2}{*}{$\frac{P_{\text {thick }}}{P_{\text {thin }}}$} & \multicolumn{2}{|c|}{$\overline{\tau_{1031.9}}$} \\
\hline & $1175.7 \AA$ & $1174.9 \AA$ & & & Hom. & Inh. & $1031.9 \AA$ & $1037.6 \AA$ & & & Hom. & Inh. \\
\hline Quiet & $6.01(0.20)$ & $2.68(0.18)$ & $2.24(0.17)$ & 0.74 & 1.55 & 0.67 & $26.83(0.20)$ & $13.75(0.19)$ & $1.95(0.03)$ & 0.97 & 0.18 & 0.08 \\
\hline $\mathrm{F} \mathrm{TOT}^{a}$ & $5.69(0.26)$ & $3.30(0.25)$ & $1.72(0.15)$ & 0.57 & 3.82 & 1.32 & $8.86(0.32)$ & $5.29(0.72)$ & $1.67(0.24)$ & 0.84 & 1.13 & 0.50 \\
\hline F1PK & $35.37(2.17)$ & $24.04(2.23)$ & $1.47(0.16)$ & 0.49 & 6.39 & 1.76 & $20.80(2.11)$ & $14.06(1.62)$ & $1.48(0.23)$ & 0.74 & 2.32 & 0.91 \\
\hline F2TOT & $4.33(0.24)$ & $3.17(0.24)$ & $1.37(0.13)$ & 0.45 & 9.21 & 2.02 & $1.43(0.23)$ & $0.90(0.29)$ & $1.59(0.57)$ & 0.79 & 1.65 & 0.69 \\
\hline F2PK & $28.41(1.68)$ & $23.91(1.66)$ & $1.19(0.11)$ & 0.39 & 14.5 & 2.52 & $20.30(2.05)$ & $13.61(1.58)$ & $1.49(0.23)$ & 0.75 & 2.17 & 0.86 \\
\hline \multicolumn{13}{|l|}{ STIS } \\
\hline Quiet $^{b}$ & $7.00(0.19)$ & $2.72(0.18)$ & $2.57(0.18)$ & 0.85 & 0.76 & 0.35 & & & & & & \\
\hline Quiet $^{c}$ & $6.35(0.19)$ & $2.71(0.17)$ & $2.34(0.16)$ & 0.65 & 2.49 & 0.98 & & & & & & \\
\hline Flare & $5.10(0.18)$ & $3.07(0.17)$ & $1.65(0.11)$ & 0.55 & 4.29 & 1.42 & & & & & & \\
\hline Fl PK & $9.46(0.49)$ & $5.80(0.48)$ & $1.63(0.16)$ & 0.54 & 4.55 & 1.47 & & & & & & \\
\hline
\end{tabular}

${ }^{a}$ Flare total and Peak emission abbreviated as TOT and PK, respectively. ${ }^{b}$ STIS O6JG. ${ }^{c}$ STIS O61S.

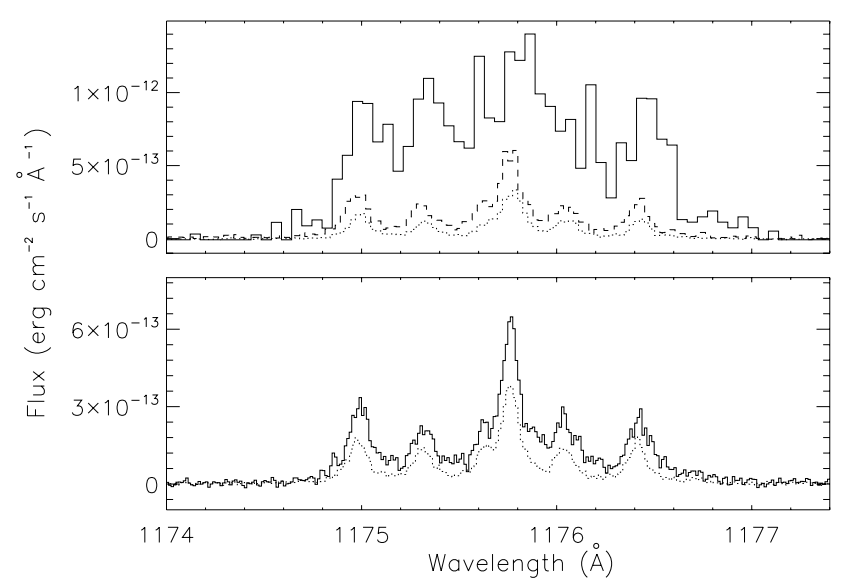

Fig. 2. STIS and FUSE C III $1176 \AA$ multiplet spectra. The top panel shows the FUSE spectra: including the spectrum from the flare peak (solid line), total flare (dashed line), and quiescence (dotted line). The lower panel shows the STIS spectra for the total flare (solid line) and quiescence (dotted line). (Note these spectra were not obtained simultaneously.)

set to be equal for all lines, but the actual values were left as free parameters.

\section{Results}

\subsection{Opacity derived using line ratios}

The ratio of the C III 1175.7 to $1174.9 \AA$ line fluxes is proportional to the ratio of their Einstein $A$ values, and is expected to be 3 under optically thin conditions. SUMER observations have shown this ratio approaches the optically thin value near the Sun's center, but deviates significantly for path length approaching the solar limb (Brooks et al. 2002). The SUMER results are consistent with the earlier results of Doyle \& McWhirter (1980) using Skylab data. For AD Leo, the observed C III ratios show deviations from the optically thin approximation in all spectra, both flaring and quiescent. We show the C III $1176 \AA$ spectra for the FUSE quiescent, total flare 1 and flare 1 peak spectra and the STIS quiescent and flare spectra in Fig. 2. The quiescent spectrum has a $\mathrm{C}$ III ratio of $2.24 \pm 0.17$, while the spectra extracted from the flare peaks show the largest deviations from the optically thin case, with ratios of $1.47 \pm 0.16$ and $1.19 \pm$ 0.11 for flare 1 and 2 peaks, respectively. In Table 1 the $C$ III and $\mathrm{O}$ VI fluxes and line ratios are presented.

The ratio of the total emission of the C III $1176 \AA$ multiplet to that of the C III $977 \AA$ line has a density dependence in the $10^{8}-5 \times 10^{10} \mathrm{~cm}^{-3}$ range. We calculated a C III $\lambda 1176 / \lambda 977 \mathrm{ra}-$ tio of $1.3 \pm 0.10$ for the quiescent spectrum and $>1.5$ for all flaring spectra. Using CHIANTI (Dere et al. 1997) to calculate theoretical line ratios indicates $n_{\mathrm{e}}>10^{11} \mathrm{~cm}^{-3}$ for the quiescent, total flare and flare peak spectra. However, as pointed out in previous work (Christian et al. 2004), the C III $977 \AA$ emission line is optically thick, and any diagnostics that use this line provide only a lower limit to the density. We make use of other diagnostic lines to derive the electron density in the next section. However, we can estimate the amount of flux lost from the $1176 \AA$ multiplet by scaling from the $1174.93 \AA$ line, which we assume to be optically thin. Under optically thin conditions the $1174.93 \AA$ line contributes about $14 \%$ of the total $1176 \AA$ multiplet flux, and as the optical depth increase its relative contribution increases. We find that $80 \%$ of the expected C III 1176 flux is observed during quiescence, while $70 \%$ and $80 \%$ of the expected flux is observed for flares 1 and 2, respectively.

The FUSE OVI 1032 and $1038 \AA$ resonance lines and STIS N V, Si IV and C IV resonance lines also provide constraints on the transition region scale heights. For the optically thin case, the ratio of their line fluxes should be 2 . There is no strong evidence for significant opacity in the quiescent spectrum with an observed O VI ratio of $1.95 \pm 0.03$. However, the flares show a modest amount of opacity with the flare 1 and flare 2 peak spectra showing similar ratios of $1.48 \pm 0.23$. Similarly, the observed $\mathrm{N} v$ quiescent spectrum shows no deviation from the optically thin case with a ratio of $2.20 \pm 0.06$, and the $\mathrm{N} V$ flare and flare peak spectra are optically thin within the derived uncertainties. Deviations from optically thin conditions are detected for the STIS flare spectra for Si IV and C IV in the quiescent spectra and flaring spectra. The Si IV and C IV flare peak spectra show the greatest opacity, with ratios of $1.49 \pm 0.21$ and $1.61 \pm 0.18$, respectively. STIS line fluxes and derived ratios are summarized for the quiescent and flaring spectra in Table 2. Comparison of the FUSE and STIS spectra of the resonance lines is presented in the next section. 
Table 2. Line fluxes and ratios of the C IV, Si IV, and N V doublets observed with STIS. The fluxes are given in units of $10^{-14} \mathrm{erg} \mathrm{cm}^{-2} \mathrm{~s}^{-1}$ followed by the estimated error in brackets. The fluxes given for each of the flares are the total observed fluxes minus the quiescent components.

\begin{tabular}{|c|c|c|c|c|c|c|}
\hline \multirow[t]{2}{*}{ Ion } & \multirow{2}{*}{\multicolumn{2}{|c|}{ Flux }} & \multirow[t]{2}{*}{ Ratio } & \multirow{2}{*}{$\begin{array}{l}\frac{P_{\text {thick }}}{P_{\text {thin }}} \\
\end{array}$} & \multicolumn{2}{|c|}{$\tau_{\text {ion }}$} \\
\hline & & & & & Hom. & Inh. \\
\hline $\mathrm{NV}$ & 1238.7 & 1242.8 & & & \multicolumn{2}{|c|}{$\tau_{1238.7}$} \\
\hline O61S Quiet & $9.0(0.14)$ & $4.1(0.1)$ & $2.20(0.06)$ & 1.10 & ... & $\ldots$ \\
\hline Flare & $2.22(0.14)$ & $1.24(0.13)$ & $1.79(0.21)$ & 0.89 & 0.71 & 0.33 \\
\hline Flare Peak & $4.05(0.62)$ & $2.19(0.37)$ & $1.85(0.42)$ & 0.92 & 0.49 & 0.23 \\
\hline O6JG Quiet & $8.8(0.17)$ & $4.1(0.52)$ & $2.14(0.26)$ & 1.07 & $\ldots$ & $\ldots$ \\
\hline Si IV & 1393.8 & 1402.8 & & & \multicolumn{2}{|c|}{$\tau_{1393.8}$} \\
\hline O61S & $8.73(0.19)$ & $4.86(0.10)$ & $1.79(0.05)$ & 0.90 & 0.63 & 0.30 \\
\hline Flare & $8.58(0.19)$ & $5.57(0.4$ & $1.54(0.14)$ & 0.77 & 1.90 & 0.77 \\
\hline Flare Peak & $16.15(0.69)$ & $10.84(1.46)$ & $1.49(0.21)$ & 0.75 & 2.17 & 0.86 \\
\hline O6JG Quiet & $8.9(0.18)$ & $4.70(0.15)$ & $1.89(0.07)$ & 0.95 & 0.29 & 0.14 \\
\hline CIV & 1548.2 & 1550.8 & & & \multicolumn{2}{|c|}{$\tau_{1548.2}$} \\
\hline O61S Quiet & $30.1(0.4)$ & $17.8(0.3)$ & $1.69(0.04)$ & 0.84 & 1.10 & 0.50 \\
\hline Flare & $22.41(0.79)$ & $13.42(0.49)$ & $1.67(0.09)$ & 0.83 & 1.23 & 0.54 \\
\hline Flare Peak & $43.84(2.42)$ & $27.24(2.73)$ & $1.61(0.18)$ & 0.80 & 1.54 & 0.65 \\
\hline O6JG Quiet & $30.0(0.62)$ & $17.8(0.38)$ & $1.68(0.05)$ & 0.84 & 1.10 & 0.50 \\
\hline
\end{tabular}

\subsection{Line ratio constraints on plasma emission size}

We can now use the derived line flux ratios to estimate the escape probabilities for our lines of study, and from these and an assumed geometry we can then estimate each line's optical depth. For pairs of lines arises from a common upper level (C III 1175.71 $\AA$ and $1174.94 \AA$ in the present study), the ratio of their fluxes is the ratio of their Einstein $A$-values reduced by the ratio of photon escape probabilities (see Eq. (2) in Bloomfield et al. 2002). Pairs of lines arising in a common lower level (O VI, $\mathrm{N} \mathrm{V}, \mathrm{Si}$ IV and C IV in the present study), the ratio of their fluxes scales as the ratio of their oscillator strengths reduced by the photon escape probability (see Eq. (4) in Bloomfield et al. 2002). For each of these two categories of line pairs, we consider both homogeneous and inhomogeneous distributions of emitters and absorbers (Kastner \& Kastner 1990; Bloomfield et al. 2002). The emitters and absorbers are spatially distinct in the inhomogeneous case. The analytic form of the escape probabilities is given in Bloomfield et al. (2002) Eqs. (5) and (6) for the homogeneous case and Eqs. (7) and (8) for the inhomogeneous one. We present our optical depth for AD Leo derived from each of these distributions in Table 1 , and note that the values from the homogeneous approximation are a factor of 2-3 times larger than those from the inhomogeneous distribution. We now use these optical depths to constrain the extent of the scattering region for both the homogeneous and inhomogeneous distributions.

With estimates of the optical depth we can use Eq. (1) to derive information on the extent of the scattering region with the only unknown quantity being the electron density. We emphasize that Eq. (1) has been derived on the assumption of thermal broadening only. If a non-thermal contribution is included, the optical depths would decrease and hence the derived path lengths would decrease. There is currently little information on the non-thermal broadening in stellar transition regions (Redfield et al. 2002) but if solar values are assumed the path lengths could decrease by up to a factor of 3 . As mentioned above, the C III $977 \AA$ emission line is optically thick, and does not provide an accurate estimate of density. However, a powerful density diagnostic are the O IV lines near $1400 \AA$, in particular the O IV $\lambda 1399.78 / \lambda 1404.79$ and $\lambda 1407.38 / \lambda 1404.79$ ratios formed around $\log (T)=5.1$. These are available from the
STIS observations in the same echelle order as Si IV, and we have derived both O IV ratios for both STIS quiescent and the total flare spectra (with a better signal to noise than the flare peak spectra). The O IV $\lambda 1399.78 / \lambda 1404.79$ ratio $\left(R_{1}\right)$ has values of $0.96 \pm 0.11$ and $1.20 \pm 0.20$ for the quiescent and flaring, respectively. Similarly, the $\lambda 1407.38 / \lambda 1404.79$ ratio $\left(R_{2}\right)$ is $1.06 \pm 0.13$ and $1.15 \pm 0.23$ for the quiescent and flaring, respectively. The O IV $R_{1}$ and $R_{2}$ quiescent values imply densities of $3 \times 10^{10} \mathrm{~cm}^{-3}$ and $4.5 \times 10^{10} \mathrm{~cm}^{-3}$ (Keenan et al. 2002), and we therefore adopt a quiescent plasma density of $4 \times 10^{10} \mathrm{~cm}^{-3}$. For the flare, the $R_{1}$ and $R_{2}$ ratios both imply densities of $\approx 6 \times$ $10^{10} \mathrm{~cm}^{-3}$ and we therefore adopt this value.

We show a comparison of the observed FUSE and STIS spectra and the CHIANTI model for the C III multiplet in Fig. 3. The CHIANTI models were derived with the above densities and for a solar flare differential emission measure (DEM). We also show a comparison of the theoretical line profiles and the observed FUSE O VI and STIS N V, Si IV, and C IV spectra in Fig. 4. Line profiles were generated with the CHIANTI spectral synthesis package for the above derived density, and a solar flare DEM for the flare spectra. The opacity effects for the C III $1175.71 \AA$ and C IV 1548.2 $\AA$ lines are clearly visible in the figures. The observed profiles also show clear redshifted components. These components were accounted for in the spectral fitting to derive the line fluxes, but are not discussed here. The redshifted components were discussed in detail for AD Leo by Hawley et al. (2003).

Using the electron density estimates from the OIV line ratios and the optical depths we can now derive values for the effective size of the scattering region (Eq. (1)) for both quiescent and flare spectra, and for both homogeneous (Hom) and inhomogeneous (Inh) source geometries. For the quiescent FUSE C III spectra, we find path lengths Hom $_{\text {CIII }} \approx 36 \mathrm{~km}$ and $\mathrm{Inh}_{\mathrm{CIII}} \approx 16 \mathrm{~km}$ for homogeneous and inhomogeneous geometries, respectively. The STIS values for the C III path lengths are approximately 50\% larger than those from FUSE. This may be due to AD Leo being slightly more active in its quiescent state during the STIS observations and possibly also because our STIS quiescent spectrum may be contaminated with several smaller flares. The O6JQ observation has a path length about $50 \%$ of the derived FUSE values. For the flaring 


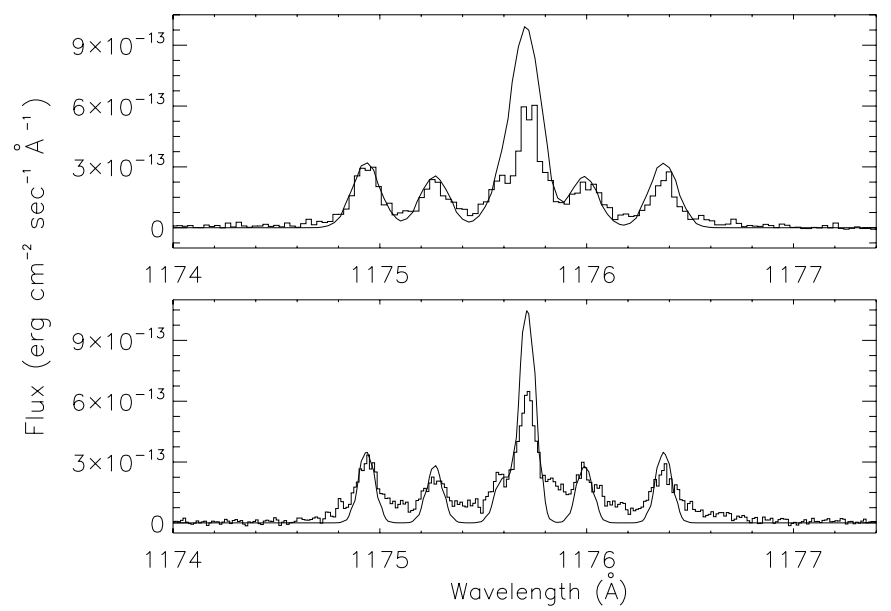

Fig. 3. Shown are the theoretical line profiles (solid line) over-plotted on the observed spectra (solid histogram). The top panel show the FUSE C III $1176 \AA$ A spectrum during flare 1, and the lower panel show the STIS C III spectrum also during a flare. The line profiles were generated with CHIANTI using our derived density $\left(6 \times 10^{10} \mathrm{~cm}^{-3}\right)$ and solar flare DEMs for both panels (please see text). The theoretical profiles are scaled to match the observed flux from the optically thin $1174.9 \AA$ line.

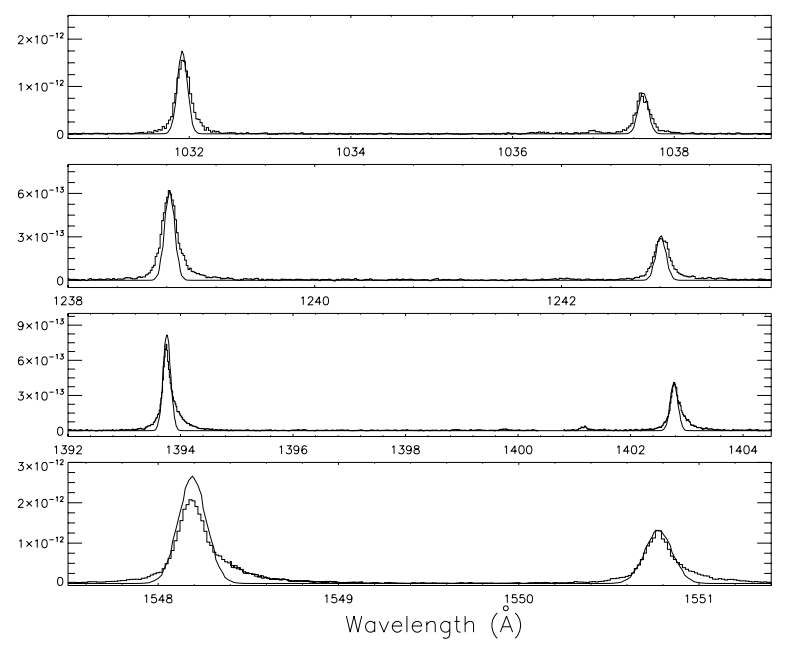

Fig. 4. Shown are the theoretical line profiles (solid line) over-plotted on the observed spectra (solid histogram). The top panel show the FUSE O VI spectrum during flare 1, and the lower panels show the STIS NV, Si IV and C IV spectrum during the flare. The line profiles were generated with CHIANTI using our derived density $\left(6 \times 10^{10} \mathrm{~cm}^{-3}\right)$ and solar flare DEMs (please see text). As in Fig. 3, the theoretical profiles are scaled to match the observed flux from the optically thin components, each occurring at longer wavelengths.

FUSE C III spectra, we find Hom $_{\text {CIII }} \approx 65 \mathrm{~km}$ and $\mathrm{Inh}_{\mathrm{CIII}} \approx$ $22 \mathrm{~km}$ path lengths for homogeneous and inhomogeneous geometries for flare 1, respectively, and the spectra extracted from the flare 1 peak also show similar values. The derived path lengths for flare 2 are about $50 \%$ larger, but again the flare and flare peak are in agreement. The STIS C III flare spectra give very similar path lengths to those derived for the FUSE flare spectra. The path lengths are summarized in Table 3.

STIS also provided several other resonance line opacity measurements, namely N V, Si IV, and C IV. The flare spectra for all three ions show strong opacity effects and deviate from the optically thin approximation. The extent of the scattering region derived from the C IV and Si IV flare spectra are similar, with Hom $_{\mathrm{CIV}} \approx 50 \mathrm{~km}$ and $\mathrm{Inh}_{\mathrm{CIV}} \approx 20 \mathrm{~km}$. During quiescence,
Table 3. Table of derived path lengths for observed ions.

\begin{tabular}{lll}
\hline \hline Ion & \multicolumn{2}{l}{ Path length (km) } \\
& Hom. & Inh. \\
\hline O vI & & \\
...Quiet & 24 & 11 \\
...Flare 1 & 109 & 48 \\
...Flare 1 Peak & 224 & 88 \\
..Flare 2 & 160 & 67 \\
C III & & \\
... Quiet & 36 & 16 \\
..Quiet (STIS) & 58 & 23 \\
...Flare (STIS) & 73 & 24 \\
..Flare1 & 65 & 22 \\
...Flare 1 peak & 66 & 18 \\
...Flare2 & 156 & 34 \\
C IV & & \\
...Quiet (STIS) & 62 & 28 \\
...Flare (STIS) & 50 & 22 \\
Si IV & & \\
...Quiet (STIS) & 24 & 11 \\
...Flare (STIS) & 52 & 21 \\
\hline
\end{tabular}

Table 4. Comparison of path lengths.

\begin{tabular}{|c|c|c|c|c|}
\hline \multirow[t]{3}{*}{ Star } & \multicolumn{4}{|c|}{ Path length $(\mathrm{km})$} \\
\hline & \multicolumn{2}{|c|}{ Quiet } & \multicolumn{2}{|c|}{ Flare } \\
\hline & Hom. & Inh. & Hom. & Inh. \\
\hline \multicolumn{5}{|l|}{ O VI } \\
\hline $\mathrm{AU} \mathrm{Mic}^{a}$ & 10 & 5 & & \\
\hline Prox Cen ${ }^{b}$ & $\ldots$ & $\ldots$ & 380 & 120 \\
\hline AD Leo & 24 & 11 & $100-200$ & $48-200$ \\
\hline \multicolumn{5}{|l|}{ C III } \\
\hline $\operatorname{Sun}^{c}$ & 50 & $\ldots$ & $\ldots$ & $\ldots$ \\
\hline AU Mic & 20 & 7 & 100 & 20 \\
\hline Prox Cen & 13 & 6 & $24-80$ & $8-25$ \\
\hline AD Leo & 36 & 16 & 60-160 & $12-34$ \\
\hline \multicolumn{5}{|l|}{ C IV } \\
\hline $\mathrm{YZ} \mathrm{CMi}^{d}$ & $\ldots$ & 5 & $\ldots$ & $\ldots$ \\
\hline Prox Cen & 30 & 15 & $\ldots$ & $\ldots$ \\
\hline AD Leo & 62 & 28 & 50 & 22 \\
\hline
\end{tabular}

${ }^{a}$ AU Mic results from Bloomfield et al. (2002). ${ }^{b}$ Prox Cen results from Christian et al. (2004). ${ }^{c}$ Solar results from Doyle \& McWhirter (1980). ${ }^{d}$ YZ CMi results from Mathioudakis et al. (1999).

the CIV and SiIV ratios show significant opacity, but the $\mathrm{NV}$ lines show no opacity effects. The C IV path lengths are $\mathrm{Hom}_{\mathrm{CIV}} \approx 60 \mathrm{~km}$ and $\mathrm{Inh}_{\mathrm{CIV}} \approx 30 \mathrm{~km}$, while Si IV indicates Hom $_{\text {SiIV }} \approx 24 \mathrm{~km}$ and $\mathrm{Inh}_{\mathrm{SiIV}} \approx 10 \mathrm{~km}$. These values are also summarized in Table 3.

\section{Discussion}

During the flaring activity of AD Leo observed with FUSE the optical depths increased by factors of 6-9. Although this change in optical depth does increase the physical extent of the scattering layer, as we adopted a 50\% higher density for the flare spectra, the flare scattering layers have similar sizes to those derived from the quiescent spectra. We compare the path lengths from our current results for AD Leo to those of the Sun, AU Mic (Bloomfield et al. 2002), YZ CMi (Mathioudakis et al. 1999), and Prox Cen (Christian et al. 2004) in Table 4. We find the quiescent path lengths for OVI are about a factor of 2 larger for $\mathrm{AD}$ Leo than AU Mic. The AD Leo quiescent path lengths for C III are approximately twice those of AU Mic and a factor of 
three larger than those of Prox Cen. Similarly the C IV results for AD Leo in quiescence are a factor of 2 larger than Prox Cen and three times larger than YZ CMi for the inhomogeneous case. AD Leo, with $\log \left(L_{\mathrm{X}} / L_{\mathrm{bol}}\right)$ of -3 , is much more active than Prox Cen $\left(\log \left(L_{\mathrm{X}} / L_{\mathrm{bol}}\right) \approx-3.8\right)$ and about $50 \%$ more active than YZ CMi $\left(L_{\mathrm{X}} / L_{\mathrm{bol}} \approx-3.3\right)$. AU Mic has a similar activity level, but had a $\mathrm{C}$ III peak flare flux about 3 times smaller than those observed for AD Leo, and this higher flux may explain the much larger path lengths for the latter.

\section{Conclusions}

We study the effects of opacity in the transition region of the active, late-type star AD Leonis. As shown previously, opacity can be used as a diagnostic tool, since the optical depth is proportional to the line of sight path length. Hence, optical depths combined with independent measurements of the electron density allow direct estimates of the path lengths in the transition region. The $\mathrm{C}$ III lines show significant opacity during both the quiescent and flaring states of $\mathrm{AD}$ Leo, with up to $30 \%$ of the expected flux being lost in the latter. Analysis of other FUV resonance lines also showed opacity effects, but with $\approx 20 \%$ of the expected flux being lost. For AD Leo discussed here, we find path lengths during quiescence in the range of $\approx 20-60 \mathrm{~km}$ and $\approx 10-30 \mathrm{~km}$ for homogeneous and inhomogeneous geometries, respectively. Path lengths derived during flaring were $\approx 2-3$ times larger. These path lengths are very similar to those found for other active, late-type stars and that of the solar transition region (Keenan \& Kingston 1986; Doschek et al. 2004). Therefore, solar and stellar transition regions must have similar spatial characteristics, and it may be fruitful to compare transition region sizes for hotter stars further up the main-sequence.
Acknowledgements. This research was partially supported by a NASA FUSE Guest-Investigator Grant (NNG04GC75G). F.P.K. is grateful to AWE Aldermaston for the award of a William Penney Fellowship. Data sets presented here were obtained from the Multimission Archive at the Space Telescope Science Institute (MAST). STScI is operated by the Association of Universities for Research in Astronomy, Inc., under NASA contract NAS5-26555. Support for MAST for non-HST data is provided by the NASA Office of Space Science via grant NAG5-7584 and by other grants and contracts.

\section{References}

Brooks, D. H., Fischbacher, G. A., Fludra, A., et al. 2000, A\&A, 357, 697 Bloomfield, D. S., Mathioudakis, M., Christian, D. J., Keenan, F. P., \& Linsky, J. 2002, A\&A, 390, 219 (Paper I)

Christian, D. J., Mathioudakis, M., Bloomfield, D. S., Dupuis, J., \& Keenan, F. P. 2004, ApJ, 612, 1140

Dere, K. P., Landi, E., Mason, H. E., Monsignori-Fossi, B. C., \& Young, P. R. 1997, A\&AS, 125, 149

Doschek, G. A., \& Feldman, U. 2004, ApJ, 601, 1061

Doyle, J. G., \& McWhirter, R. W. P. 1980, MNRAS, 193, 947

Favata, F., Micela, G., \& Reale, F. 1999, A\&A, 354, 1021

Hawley, S. L., Allred, J. C., Johns-Krull, C. M., et al. 2003, ApJ, 597, 535

Jordan, C. 1967, Sol. Phys., 2, 441

Kastner, S. O., \& Kastner, R. E. 1990, J. Quant. Spec. Radiat. Transf., 44, 275

Keenan, F. P., \& Kingston, A. E. 1986, MNRAS, 2220, 493

Keenan, F. P., Ahmed, S., Brage, T., et al. 2002, MNRAS, 337, 901

Mathioudakis, M., McKenny, J., Keenan, F. P., Williams, D. R., \& Phillips, K. J. H. 1999, A\&A, 351, L23

Mitchell, A. C. G., \& Zemansky, M. W. 1961, in Resonance Radiation and Excited Atoms (Cambridge University Press)

Moos, H. W., Cash, W. C., Cowie, L. L., et al. 2000, ApJ, 538, L1

Redfield, S., Linsky, J. L., Ake, T. B., et al. 2002, ApJ, 581, 626

Sahnow, D. J., Moos, H. W., Ake, T. B., et al. 2000a, ApJ, 538, L7

Sahnow, D. J., Moos, H. W., Ake, T. B., et al. 2000b, in UV, Optical, and IR Space Telescopes and Instruments Proc. SPIE, ed. J. B. Breckinridge, \& P. Jakobsen, 4013, 334

Sciortino, S., Maggio, A., Favata, F., \& Orlando, S. 1999, A\&A, 342, 502 van Dixon, W., Kruk, J., \& Murphy, E. 2001, Calfuse Reference Guide, http://fuse.pha.jhu.edu/analysis/calfuse.html 